\title{
INVESTIGACIÓN
}

\section{ESTRATIFICACIÓN DEL RIESGO DE PIE DIABÉTICO EN ADULTOS CON DIABETES MELLITUS}

\author{
STRATIFICATION OF DIABETIC FOOT RISK IN ADULTS WITH DIABETES MELLITUS
}

SANTOS-FLORES, JESÚS MELCHOR*, SANTOS-FLORES IZAMARA*, GEVARA-VALTIER MILTON CARLOS**,

GUTIÉRREZ-VALVERDE JUANA MECEDES***, RUVALCABA-RODRÍGUEZ, MARÍA DIANA****

\section{RESUMEN}

ntroducción: El pie diabético es una de las complicaciones más graves de la diabetes mellitus, esta constituye una de las enfermedades crónicas degenerativas de mayor importancia en México, dado que existen 6.4 millones de diabéticos lo que representa a un $9.2 \%$ de la población adulta, mismos que están expuestos a desarrollar complicaciones de esta naturaleza. Objetivo: El objetivo de este estudio fue conocer y estratificar el riesgo de pie diabético en personas con Diabetes Mellitus Tipo 2 de acuerdo a edad y sexo, hospitalizadas en una Institución de segundo nivel en Sabinas Hidalgo, Nuevo León, México. Material-Métodos: El diseño fue descriptivo-transversal con una muestra 21 personas (Calculada a partir de una población de 121 personas), cada participante se seleccionó mediante muestreo aleatorio simple. La colecta de datos se llevó a cabo con la Escala para Valoración del Pie Diabético con un alfa de Cronbach de 0.74. Resultados: Los resultados se analizaron con el paquete estadístico SPSS versión 23 para Windows 10. Se encontró que el 41.9\% de los pacientes presentan Riesgo Moderado, seguidos de los que tenían Riesgo Alto (38.1\%); así mismo, los hombres y quienes tenían entre 30 y 44 años fueron los que denotaron peores indicadores que los ubicaba en Riesgo Moderado. Se requieren estudios posteriores que incluyan variables bioquímicas y cognitivas desde una perspectiva ecológica.

\section{Palabras clave: pie diabético, diabetes mellitus, clasificación, amputación (DeCS, BIREME).}

\section{ABSTRACT}

Introduction: Diabetic foot is one of the most serious complications of diabetes mellitus; this is one of the most important chronic degenerative diseases in Mexico, since there are 6.4 million diabetics representing $9.2 \%$ of the adult population, they are exposed to develop complications of this nature. Objective: The objective of this study was to know and stratify the risk of diabetic foot in people with Type 2 Diabetes Mellitus according to age and sex, hospitalized in a second level institution in Sabinas Hidalgo, Nuevo Leon, Mexico. Material-Methods: The design was descriptive-transversal with a sample of 21 people (calculated from a population of 121 people), each participant was selected by simple random sampling. The data collection was carried out with the Diabetic Foot Assessment Scale with a Cronbach's alpha of 0.74. Results: The results were analyzed with the statistical package SPSS version 23 for Windows 10 . It was found that $41.9 \%$ of the patients presented Moderate Risk, followed by those with High Risk (38.1\%); likewise, men who were between the ages of 30 and 44 years old were the ones that showed the worse indicators that placed them in Moderate Risk. Further studies that include biochemical and cognitive variables from an ecological perspective are required.

Key words: diabetic foot, diabetes mellitus, classification, amputation (DeCS, BIREME).

*Licenciatura en Enfermería. Facultad de Enfermería. Universidad Autónoma de Nuevo León. Correo electrónico: santos_meme_10@hotmail.com; santos_m21@outlook.como.

** Doctorado en Educación. Facultad de Enfermería. Universidad Autónoma de Nuevo León.

***Doctorado en Ciencias de Enfermería. Universidad Autónoma de Nuevo León.

****Maestría en Ciencias de Enfermería. Universidad Autónoma de Nuevo León. 


\section{INTRODUCCIÓN}

La diabetes mellitus (DM) afecta a todos los países a todas las edades, sin importar la economía y sufraga como problema de salud pública debido a su prevalencia que va en incremento. En el año 2000, 150 millones de personas en el mundo padecían de DM y se estima que en el año 2025 incrementará a 300 millones ${ }^{(1-2)}$. Constituye un problema metabólico importante (3) la Encuesta Nacional de Salud y Nutrición (ENSANUT) identificó a 6.4 millones de diabéticos (9.2\% de la población adulta). Se registró que un 30\% de los pacientes presentan complicaciones ${ }^{(4)}$. Existen distintas complicaciones que se desarrollan durante la $\mathrm{DM}$, del total de individuos que refieren diagnóstico previo, $47.6 \%$ visión disminuida, 38\% ardor, dolor o pérdida de sensibilidad en los pies, $13.9 \%$ daños en la retina. Como complicaciones más graves son la amputación, 2\% (128 mil) reportaron amputaciones por pie diabético (PD), $1.4 \%$ diálisis, $2.8 \%$ infartos ${ }^{(5)}$.

El PD constituye uno de los problemas y complicaciones más relevantes y quizás el sitio del organismo en el que más se hace evidente el efecto devastador de las complicaciones ${ }^{(5-6)}$.

Tiene relevancia social según distintos autores, ya que ocasiona una elevada morbilidad, alta tasa de amputaciones, elevado promedio de estadía hospitalaria y altos costos hospitalarios y económicos ${ }^{(7)}$. El PD y su impacto sobre los sistemas de salud ha sido puesto de manifiesto en diferentes estudios en todos los continentes, por su complejidad etiopatogénica y clínica es una entidad interdisciplinaria en su estudio y tratamiento. La complicación más temida de la DM es el PD y como consecuencia de este la amputación de un miembro, con la carga psicológica y social que lleva aparejada ${ }^{(8)}$. Se ha evidenciado que el riesgo de amputación es 15 veces mayor que en individuos no diabéticos, y este trastorno se relaciona con 50 a $70 \%$ de las amputaciones no traumáticas que se efectúan en hospitales generales ${ }^{(3)}$. Los factores de riesgo y las complicaciones pueden ser prevenidos o minimizados a partir de un amplio conocimiento de estos y en la clasificación de los mismos, con el fin de disminuir el desarrollo de úlceras en el pie, las amputaciones y la tasa de mortalidad $(3,9,10)$.

\section{Definición de términos}

Clasificación del riesgo del pie diabético: Sistema de clasificación del pie que permite evaluar el nivel del riesgo del pie diabético, fue evaluado mediante la Escala para Valoración del Pie Diabético de Riesgo "EVPDR" (Creada por los autores). Edad: Tiempo que ha transcurrido desde el nacimiento, fue evaluada en términos de años que reportó tener al momento de la entrevista. Género: Es lo que se atribuye a las mujeres y a los hombres social y culturalmente a partir del sexo biológico, se evaluó correspondientemente al reporte verbal en términos de femenino o masculino. Pie diabético: Es una alteración clínica de base al daño neuropático e inducida por la hiperglicemia mantenida, en la que con o sin coexistencia de isquemia, y previo desencadenante traumático, produce lesión y/o ulceración del pie ${ }^{(11)}$.

\section{Objetivos del estudio}

Identificar la frecuencia y estratificación del riesgo de pie diabético en pacientes con Diabetes Mellitus en una muestra de pacientes adultos en el Hospital General "Virginia Ayala de Garza".

1.- Identificar el pie en riesgo de los pacientes con Diabetes Mellitus asistidos en el Hospital.

2.- Determinar la frecuencia del riesgo en pacientes diabéticos con pie diabético.

3.- Establecer la estratificación del riesgo de pie diabético en pacientes diabéticos.

4.- Determinar la prevalencia de los factores de riesgo.

5.- Comparar poblaciones de mayor y menor riesgo.

\section{METODOLOGÍA}

\section{Diseño del estudio}

El diseño del estudio es descriptivo-transversal, dado a que únicamente se pretendía observar, describir, documentar y comparar aspectos de una situación en particular que ocurre de manera natural (12).

\section{Población, muestreo y muestra}

Se contó con una población de 144 pacientes con diagnóstico de Diabetes Mellitus en diciembre del 2016. Para esta investigación el tamaño de la muestra a considerar fue de 21 pacientes con diagnóstico de Diabetes Mellitus, que asisten al Hospital General "Virginia Ayala de Garza" en el municipio de Sabinas Hidalgo Nuevo León. Usando el muestreo piloto quienes debieron cumplir con los criterios de inclusión considerados a continuación.

\section{Criterios de inclusión}

1.- Pacientes mayores de 18 años de edad.

2.- Adultos con patología diabética. 
3.- Que voluntariamente y por escrito aceptaron participar en el estudio.

\section{Instrumentos de medición}

Para obtener la respuesta de la primera pregunta de investigación se utilizó el instrumento de Escala para Valoración del Pie Diabético de Riesgo "EVPDR" (Creada por los autores), para determinar el riesgo de desarrollar pie diabético en pacientes con DM. La Escala para Valoración del Pie Diabético de Riesgo "EVPDR" es una clasificación para valorar el riesgo del pie diabético, definida así por sus autores, presenta 4 subconjuntos: Valoración Dermatológica, Valoración Neurológica, Valoración Vascular y Valoración Osteoarticular. Consta de un apartado de identificación, su estratificación es de bajo riesgo 0-5 puntos, mediano riesgo 6-13 puntos, alto riesgo $\geq 14$ puntos, cada subconjunto tiene puntajes de 0 a 2, que van desde $0=$ Normal hasta $2=$ Anormal, $0=$ No hasta $2=$ Sí, entre otros.

En cada riesgo clasificado se otorga el puntaje y se marca el color del pie en la valoración, verde para bajo riesgo, amarillo para mediano riesgo y rojo para alto riesgo. Con un alfa de Cronbach de 0.74 analizado con SPSS versión 23 , según ${ }^{(13)}$ la puntuación se encuentra entre buena ${ }^{(8)} y$ aceptable ${ }^{(7)}$.

\section{Consideraciones éticas}

El presente estudio se apegó al Reglamento de la Ley General de Salud en Materia de Investigación para la Salud. Según lo estipulado en el Título Segundo, Capítulo I, De los Aspectos Éticos de la Investigación en Seres Humanos (14). De acuerdo al artículo 13, En toda investigación en la que el ser humano sea sujeto de estudio, deberá prevalecer el criterio del respeto a su dignidad y la protección de sus derechos y bienestar.

Artículo 14, Fracciones I, V, IV, prevaleció siempre la probabilidad de los beneficiados esperados sobre los riesgos predecibles, VI, VII y VIII se contó con el consentimiento informado y por escrito de los participantes, previo a la aplicación de los instrumentos y/o realización de cualquier procedimiento. El consentimiento informado se formuló por escrito y se solicitó la firma de dos testigos y fue aprobado por la Comisión de Ética de la Institución Hospitalaria tal como lo estipula el Artículo 22, Fracciones II y IV.

En cumplimiento al Artículo 16, se protegió la privacidad de los participantes y la información obtenida se resguardó en una oficina a la cual solo tuvo acceso el equipo de investigación.

En el Artículo 17, Fracción II, la investigación se consideró de riesgo mínimo ya que se empleó la recogida de datos a través de procedimientos comunes en exámenes físicos o psicológicos de diagnósticos o tratamiento rutinarios.
Respecto al Artículo 18, el sujeto de estudio podía suspender de inmediato su participación en el estudio si así lo deseaba.

En el Artículo 20, el consentimiento informado fue el acuerdo por escrito, mediante el cual el sujeto de investigación autorizó su participación en la investigación, concordando y en respuesta al Artículo 21 Fracciones I, II, VI, VII y VIII, para hacer existente el consentimiento informado se brindó información clara y completa en donde se incluyó la justificación y objetivos, procedimientos, propósito, riesgos esperados, beneficios, garantizándole la confidencialidad de la información y la libertad de retirarse del estudio si así lo hubiera decidido.

\section{Análisis de datos}

Los datos se analizaron con el uso del paquete estadístico Statistical Package for the Social Sciences (SPSS) versión 23 para Windows 10, se utilizó estadística descriptiva y se recurrió al empleo de la media, desviación estándar, así como el uso de frecuencias y porcentajes, lo anterior para dar respuesta al objetivo de investigación.

\section{RESULTADOS}

Participaron 21 pacientes diagnosticados con Diabetes Mellitus Tipo II. La edad osciló entre 30 y 92 años, edad promedio fue 52 años ( $D E=0.740)$, respecto al género el $66.7 \%(D E=0.483)$ fueron de sexo femenino. El IMC con más frecuencia fue Obesidad I (30-34.9) con 38.1\% ( $D E=$ 1.271). De acuerdo a los factores de riesgo, el $95.2 \%$ ( $D E=$ 0.964 ) refirieron tener antecedentes heredofamiliares, el $81 \%$ presentaba sobrepeso u obesidad y el $19 \%$ presentaba el hábito de fumar. Sólo un participante presentaba antecedente de amputación (tabla 1).

Respecto a los resultados de cada subconjunto e ítem de la escala EVPDR, en dermatológico se identificó el $66.7 \%$ $(D E=0.470)$ presentaba palidez, un $57.1 \%(D E=0.507)$ presentaba piel gruesa, un $47.6 \%(D E=0.510)$ piel seca, 9.5\% $(D E=0.307)$ grietas en la piel, $52.4 \%(D E=0.853)$ callosidades. En el subconjunto vascular se identificó un $14.3 \%(D E=0.307)$ de pulsos disminuidos, un $23.8 \%$ $(D E=0.307)$ de llenado capilar de 3 segundos, se encontró la piel fría en un 19\% ( $D E=0.410)$, claudicación en un $19 \%(D E=0.410)$, dolor en reposo en un $28.6 \%$ $(D E=0.489)$. En el subconjunto neurológico se identificó un $38.1 \%(D E=0.444)$ de fatiga, calambres, dolor en extremidades inferiores, otro $38.1 \%(D E=0.470)$ en ardor, adormecimiento y hormigueo, al examen del Monofilamento Semmes-Weinstein un 14.3\% $(D E=0.447)$ presentó sensibilidad en 6 de 10 puntos. Lo anterior se puede observar en la tabla 2.

Para la frecuencia y el porcentaje del riesgo de pie diabético, se determinó una frecuencia para el bajo riesgo de 
Tabla 1. Datos de ficha de registro de valoración de paciente diabético

\begin{tabular}{|c|c|c|c|}
\hline & & $\bar{f}$ & $\%$ \\
\hline \multirow{4}{*}{ Edad } & 18-29 años & 0 & 0 \\
\hline & 30-44 años & 6 & 28.6 \\
\hline & 45-64 años & 10 & 47.6 \\
\hline & 65 o más & 5 & 23.8 \\
\hline \multirow{2}{*}{ Género } & Masculino & 7 & 33.3 \\
\hline & Femenino & 14 & 66.7 \\
\hline \multirow{6}{*}{ IMC } & Infrapeso $(\leq=18.4)$ & 0 & 0 \\
\hline & Normal (18.5-24.9) & 4 & 19.0 \\
\hline & Sobrepeso (25-29.9) & 5 & 23.8 \\
\hline & Obesidad I (30-34.9) & 8 & 38.1 \\
\hline & Obesidad II (35-39.9) & 1 & 4.8 \\
\hline & Obesidad III $(\geq=40)$ & 3 & 14.3 \\
\hline \multirow{7}{*}{ Factores de riesgo } & HTA & 12 & 57.1 \\
\hline & Tabaquismo & 4 & 19.0 \\
\hline & Antecedentes Familiares & 20 & 95.2 \\
\hline & Sedentarismo & 14 & 66.6 \\
\hline & Sobrepeso u Obesidad & 17 & 81.0 \\
\hline & Amputados (Pie Derecho) & 1 & 4.8 \\
\hline & Amputados (Pie Izquierdo) & 0 & 0 \\
\hline Fuente: FRVPD & & & \\
\hline
\end{tabular}

entre 3-5 de 21 con un porcentaje promedio de 19.05 , para el mediano riesgo con una frecuencia de entre 8-10 de 21 con un porcentaje promedio de 42.8 siendo este el más alto, para el alto riesgo una frecuencia de entre 7-8 de 21 con un porcentaje promedio de 35.7, siendo poco más de la tercera parte de la población (tabla 3).

Los resultados analizados demuestran que los pacientes se encuentran en alto riesgo un $35.7 \%$ con una edad promedio de 52 años con una predominancia del sexo femenino con 6 de 14 pacientes (42.9\%). Los pacientes que se encuentran en mediano riesgo con una edad promedio de entre 66 y 92 años con una predominancia del sexo masculino con 4 de 7 pacientes (57.1\%). Los resultados se aprecian en la tabla 4 y 5.

De acuerdo a cada subconjunto de valoración, dermatológico de encuentra en alto riesgo con $54.7 \%(D E=0.762)$, vascular en mediano riesgo con $47.6 \%(D E=0.695)$, neurológico en bajo riesgo con $62 \%(D E=0.915)$ y osteoarticular en bajo riesgo con 62\% ( $D E=0.710)$ (tabla 6).
En la asociación entre el IMC, el hábito de fumar y la estratificación en niveles de riesgo, el alto riesgo tuvo relación directa con el mayor IMC promedio encontrado que fue de 32.8 , al igual que el hábito de fumar con $9.5 \%$, con el porcentaje más alto para alto riesgo (tabla 7 ).

\section{DISCUSIÓN}

Se describió el nivel de pie de riesgo que presentan los pacientes atendidos en el Hospital General "Virginia Ayala de Garza", los resultados de este estudio se relacionan con algunos de los hallazgos de estudios anteriores (1516-17). Al describir el nivel del riesgo en pie diabético se identificó que el subconjunto dermatológico obtuvo el porcentaje más alto en el nivel de alto riesgo, neurológico y osteoarticular el porcentaje más alto en bajo riesgo, se obtuvo un porcentaje para cada estratificación del riesgo, bajo riesgo con 19.05, mediano riesgo 42.8 y alto riesgo 35.7, es decir, los pacientes atendidos en el Hospital General presentan un nivel de riesgo medio. Sin embargo, es importante destacar que el porcentaje más 
Tabla 2. Resultados por ítem de escala de valoración

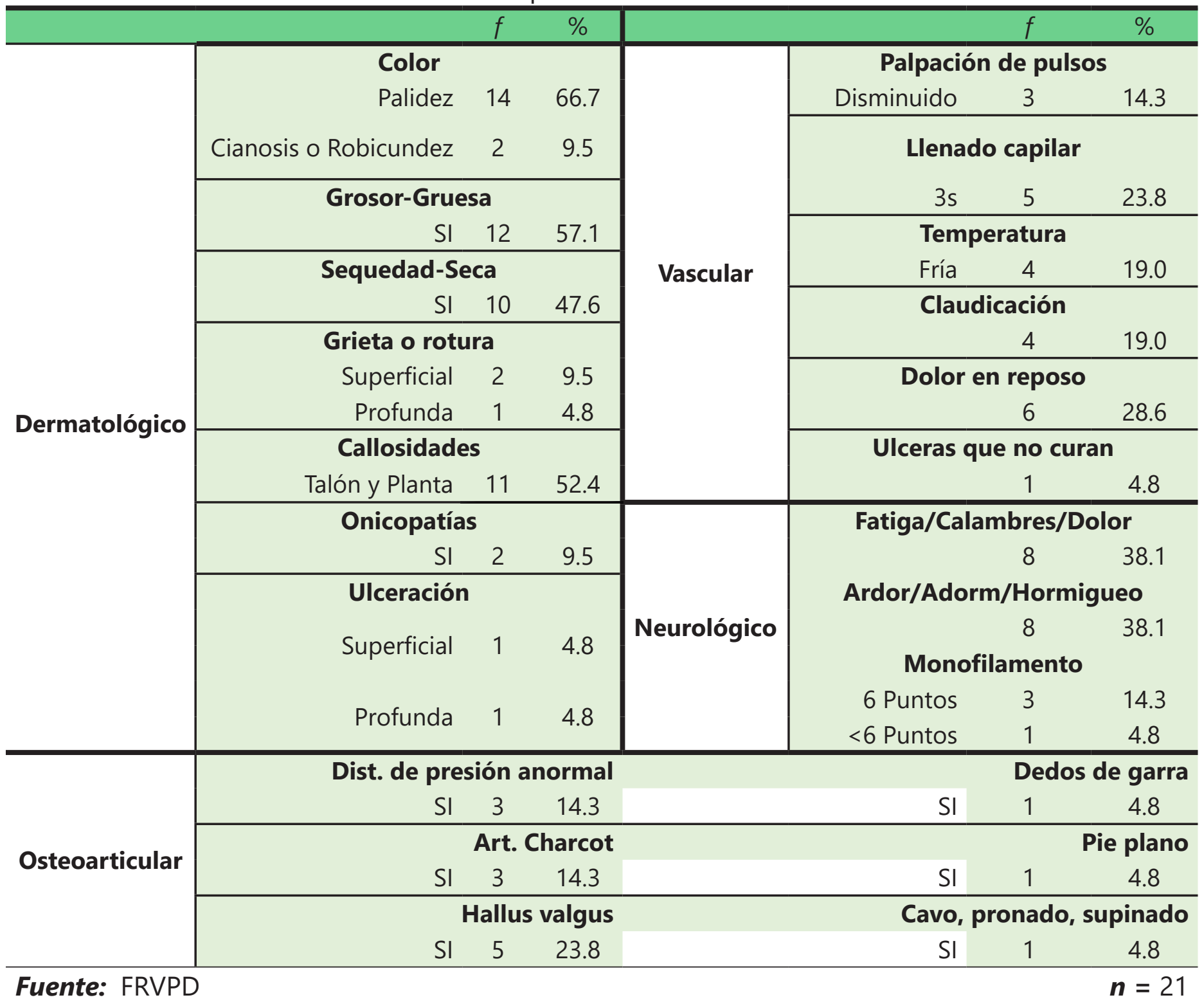

Tabla 3. Frecuencia y porcentaje del riesgo de pie diabético

\begin{tabular}{|c|c|c|c|c|c|c|c|}
\hline \multirow{2}{*}{$\begin{array}{l}\text { Pie de Riesgo/ } \\
\text { Nivel de Riesgo }\end{array}$} & \multicolumn{2}{|c|}{$\begin{array}{c}\text { 1.- Bajo Riesgo } \\
\text { (BR) }\end{array}$} & \multicolumn{2}{|c|}{$\begin{array}{l}\text { 2.- Mediano } \\
\text { Riesgo (MR) }\end{array}$} & \multicolumn{2}{|c|}{$\begin{array}{l}\text { 3.- Alto Riesgo } \\
\text { (AR) }\end{array}$} & \multirow[t]{2}{*}{$M(D E)$} \\
\hline & $\bar{f}$ & $\%$ & $f$ & $\%$ & $f$ & $\%$ & \\
\hline Pie Derecho (95.2\%) & 5 & 23.8 & 8 & 38.1 & 7 & 33.3 & $2.00(0.894)$ \\
\hline $\begin{array}{r}\text { Pie Izquierdo } \\
(100 \%)\end{array}$ & 3 & 14.3 & 10 & 47.6 & 8 & 38.1 & $2.24(0.700)$ \\
\hline \multirow{2}{*}{$\begin{array}{l}\text { Porcentaje de c/Ni- } \\
\text { vel de Riesgo }\end{array}$} & \multicolumn{2}{|c|}{ BR } & \multicolumn{2}{|c|}{ MR } & \multicolumn{2}{|c|}{ AR } & Amputado \\
\hline & \multicolumn{2}{|c|}{19.05} & \multicolumn{2}{|c|}{42.8} & \multicolumn{2}{|c|}{35.7} & 2.45 \\
\hline *Nota: & \multicolumn{2}{|c|}{$f=$ Frecuencia } & \multicolumn{2}{|c|}{$M=$ Media } & \multicolumn{3}{|c|}{$D E=$ Desviación estándar } \\
\hline
\end{tabular}


Tabla 4. Estratificación del riesgo de pie diabético de acuerdo a la edad

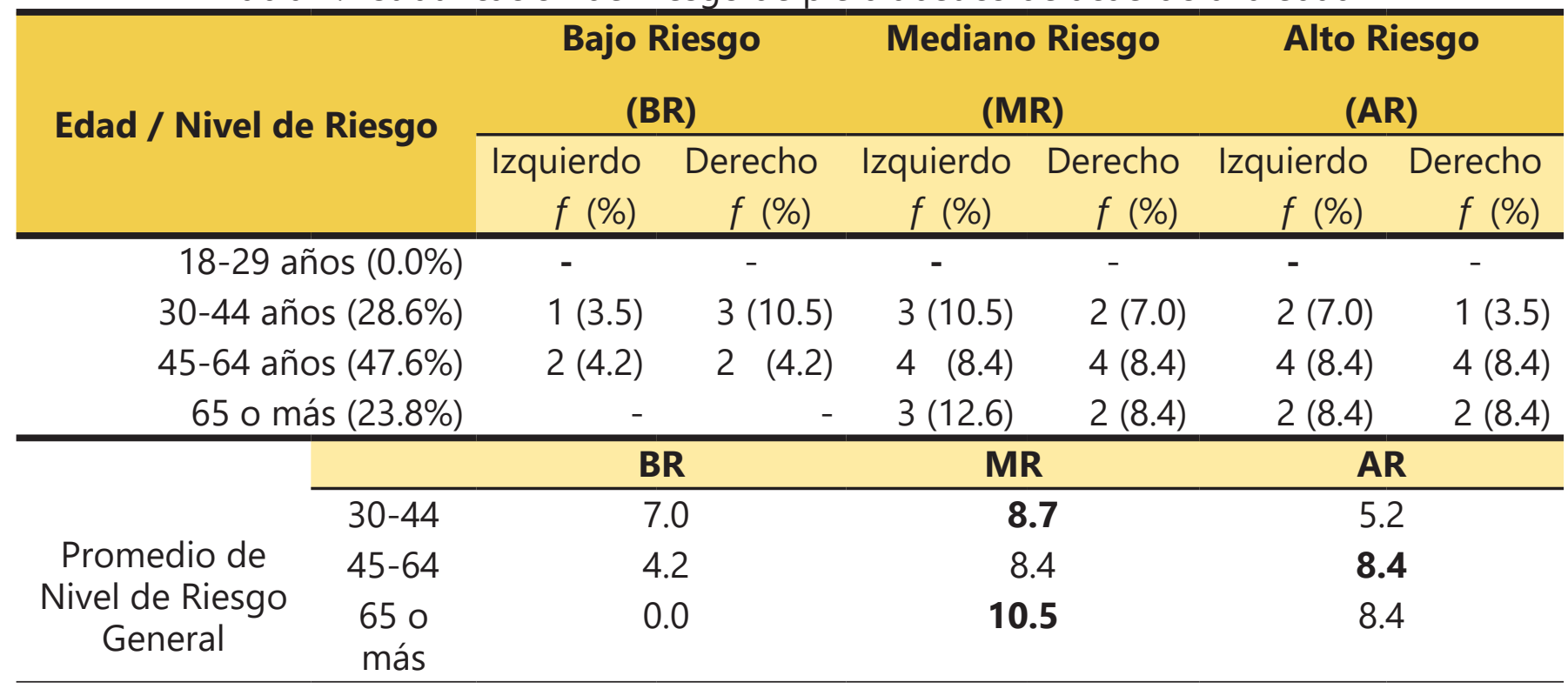

Fuente: FRVPD

$\boldsymbol{n}=21$

\begin{tabular}{|c|c|c|c|c|c|c|}
\hline \multirow{2}{*}{$\begin{array}{c}\text { Genero/ Nivel de Ries- } \\
\text { go }\end{array}$} & \multicolumn{2}{|c|}{$\begin{array}{c}\text { Bajo Riesgo } \\
\text { (BR) }\end{array}$} & \multicolumn{2}{|c|}{$\begin{array}{c}\text { Mediano Riesgo } \\
\text { (MR) }\end{array}$} & \multicolumn{2}{|c|}{$\begin{array}{c}\text { Alto Riesgo } \\
\text { (AR) }\end{array}$} \\
\hline & $\begin{array}{c}\text { Izquierdo } \\
f(\%)\end{array}$ & $\begin{array}{l}\text { Derecho } \\
f(\%)\end{array}$ & $\begin{array}{l}\text { Izquierdo } \\
f(\%)\end{array}$ & $\begin{array}{c}\text { Derecho } \\
f(\%)\end{array}$ & $\begin{array}{l}\text { Izquierdo } \\
f(\%)\end{array}$ & $\begin{array}{c}\text { Derecho } \\
f(\%)\end{array}$ \\
\hline Masculino & $1(14.3)$ & $1(14.3)$ & $4(57.1)$ & $4(57.1)$ & $2(28.6)$ & $1(14.3)$ \\
\hline Femenino & $2(14.3)$ & $4(28.6)$ & $6(42.9)$ & $4(28.6)$ & $6(42.9)$ & $6(42.9)$ \\
\hline \multirow{3}{*}{$\begin{array}{l}\text { Promedio de Nivel } \\
\text { de Riesgo General }\end{array}$} & \multicolumn{2}{|c|}{ BR } & \multicolumn{2}{|c|}{ MR } & \multicolumn{2}{|c|}{ AR } \\
\hline & Masc & Fem & Masc & Fem & Masc & Fem \\
\hline & 14.3 & 21.5 & 57.1 & 35.7 & 21.4 & 42.9 \\
\hline
\end{tabular}

bajo fue para bajo riesgo, y existe sólo una diferencia mínima de $7.1 \%$ entre mediano y alto riesgo.

El porcentaje más alto obtenido en mediano riesgo para cada subconjunto analizado en este estudio no concuerda con lo referido en la bibliografía consultada; en un estudio hecho por González ${ }^{(18)}$ con 96 sujetos se obtuvo $44.8 \%$ en riesgo 1 de 4 . En otro estudio efectuado con 625 pacientes realizado por Ibañez ${ }^{(15)}$ en España obtuvo como resultado un $51.4 \%$ en riesgo 1 de 4 . Posiblemente las diferencias en los resultados obtenidos y comparados con distintos estudios podrían atribuirse al área geográfica, cantidad de pacientes estudiados y tipo o establecimiento de salud en donde se realizó dicho estudio.

\section{Limitaciones del estudio}

Este estudio tiene limitaciones que afectan el alcance de sus resultados, y la más importante es que se realizó en un centro de segundo nivel, donde los pacientes, generalmente, acuden remitidos de sus áreas de salud, por descontrol metabólico en general y por complicaciones de la diabetes. Sin embargo, constituye un reporte completo sobre el estudio del pie de riesgo de acuerdo con las recomendaciones del documento del Consenso Internacional de Pie Diabético del año 2007, y la Guía de Referencia Rápida de Zinolox del año 2014, ofrece una perspectiva de la magnitud del problema en nuestra población, y permite identificar aquellos aspectos en los que sería oportuno profundizar para lograr una comprensión más acabada de este. 
Tabla 6. Estratificación del riesgo de pie diabético de acuerdo a subconjuntos de valoración

\begin{tabular}{|c|c|c|c|c|c|c|c|}
\hline \multirow[t]{2}{*}{$\begin{array}{l}\text { Subconjunto/ } \\
\text { Nivel de Riesgo }\end{array}$} & \multicolumn{2}{|c|}{$\begin{array}{c}\text { Bajo Riesgo } \\
\text { (BR) }\end{array}$} & \multicolumn{2}{|c|}{$\begin{array}{c}\text { Mediano Riesgo } \\
\text { (MR) }\end{array}$} & \multicolumn{2}{|c|}{$\begin{array}{c}\text { Alto Riesgo } \\
\text { (AR) }\end{array}$} & \multirow[t]{2}{*}{ M (DE) } \\
\hline & Izquierdo & Derecho & Izquierdo & Derecho & Izquierdo & Derecho & \\
\hline \multirow{5}{*}{$\begin{array}{r}\text { Dermatológico } \\
\text { Vascular } \\
\text { Neurológico } \\
\text { Osteoarticular }\end{array}$} & $f(\%)$ & $f(\%)$ & $f(\%)$ & $f(\%)$ & $f(\%)$ & $f(\%)$ & \multirow[b]{2}{*}{$2.40(0.762)$} \\
\hline & $2 \quad(9.5)$ & $2 \quad(9.5)$ & $6(28.6)$ & $8(38.1)$ & 13 (61.9) & $10(47.6)$ & \\
\hline & $8(38.1)$ & 9 (42.9) & $11(52.4)$ & $9(42.9)$ & $2(9.5)$ & $2(9.5)$ & $1.64(0.695)$ \\
\hline & $12(57.1)$ & 14 (66.7) & $3(14.3)$ & $1(4.8)$ & $6(28.6)$ & $5(23.8)$ & $1.59(0.915)$ \\
\hline & 13 (61.9) & 13 (61.9) & $6(28.6)$ & $5(23.8)$ & $2(9.5)$ & $2 \quad(9.5)$ & $1.43(0.710)$ \\
\hline \multirow{5}{*}{$\begin{array}{c}\text { Promedio de } \\
\text { Nivel de Riesgo } \\
\text { General }\end{array}$} & & BR & \multicolumn{2}{|c|}{ MR } & \multicolumn{2}{|c|}{ AR } & Riesgo \\
\hline & Derm & 9.5 & \multicolumn{2}{|c|}{33.3} & \multicolumn{2}{|c|}{54.7} & Alto \\
\hline & Vasc & 40.5 & \multicolumn{2}{|c|}{47.6} & \multicolumn{2}{|c|}{9.5} & Mediano \\
\hline & $\mathrm{Neu}$ & 62.0 & \multicolumn{2}{|c|}{9.5} & \multicolumn{2}{|c|}{26.2} & Bajo \\
\hline & Osteo & 62.0 & \multicolumn{2}{|c|}{26.2} & \multicolumn{2}{|c|}{9.5} & Bajo \\
\hline
\end{tabular}

Fuente: FRVPD

$\boldsymbol{n}=21$

Tabla 7. Asociación entre el IMC, el hábito de fumar y la estratificación en niveles de riesgo

\begin{tabular}{|c|c|c|c|c|c|c|c|}
\hline \multirow[t]{2}{*}{ Variable } & \multicolumn{2}{|c|}{$\begin{array}{c}\text { Bajo Riesgo } \\
\text { (BR) }\end{array}$} & \multicolumn{3}{|c|}{$\begin{array}{c}\text { Mediano Riesgo } \\
\text { (MR) }\end{array}$} & \multicolumn{2}{|c|}{$\begin{array}{c}\text { Alto Riesgo } \\
\text { (AR) }\end{array}$} \\
\hline & Rango & $\mathrm{M}$ & & Rango & $\mathrm{M}$ & Rangc & $\mathrm{M}$ \\
\hline \multirow[t]{3}{*}{ IMC } & $25-\geq 40$ & 27.5 & 18.5 & $-\geq 40$ & 25.1 & $25-\geq 40$ & 32.8 \\
\hline & \multicolumn{2}{|c|}{ BR } & \multicolumn{3}{|c|}{ MR } & \multicolumn{2}{|c|}{ AR } \\
\hline & \multicolumn{2}{|c|}{$f(\%)$} & \multicolumn{3}{|c|}{$f(\%)$} & \multicolumn{2}{|c|}{$f(\%)$} \\
\hline Tabaquismo & \multicolumn{2}{|c|}{$1(4.8)$} & \multicolumn{3}{|c|}{$1(4.8)$} & \multicolumn{2}{|c|}{$2(9.5)$} \\
\hline
\end{tabular}

Otra de las limitaciones importantes fue que no se encontró un instrumento que valorara la estratificación del riesgo, por tal motivo, se optó por la creación de un instrumento que se adaptara a las necesidades del Hospital General, se creó una validación de alfa de Cronbach a través del programa SPSS obteniendo un puntaje de 0.74 (entre bueno y aceptable).

\section{Recomendaciones}

Es recomendable integrar a más participantes en el estudio, al estratificar el nivel del riesgo administrar un grupo control por nivel y estructurar un programa para la atención al pie diabético en atención secundaria preventiva por tal motivo es de suma importancia que se elabore el cribado estandarizado del pie diabético. Enfocado para esto, en la inspección del pie principalmente valorar 4 áreas: dermatológico, vascular, neurológico y osteoarticular.

Los profesionales de la salud debemos ser capacitados periódicamente en el manejo del pie diabético (reconocimiento el riesgo precoz, determinación de controles periódicos según el riesgo, educación preventiva etc.) con la finalidad de mejorar las estrategias intramural y extramural.

\section{CONCLUSIÓN}

En general la detección del riesgo de lesión en el pie diabético está determinada por un alto porcentaje de pacientes con riesgo moderado y alto según la escala del test, quienes se caracterizaron por ser mayoritariamente 
de sexo femenino y con 52 años de edad promedio.

La población diabética estudiada presenta una importante prevalencia de pie de riesgo que podría aumentar a corto plazo. La presencia del riesgo en pies de pacientes diabéticos está relacionado con el sobrepeso y la obesidad, el hábito de fumar, edades avanzadas, sexo femenino, antecedentes de DM y poco cuidado dermatológico.

\section{REFERENCIAS BIBLIOGRÁFICAS}

1. FID. Plan mundial contra la diabetes. Federación internacional de diabetes FID. 2012.

2. Gavin JR 3rd, Freeman JS, Shubrook JH, Lavernia F. Type 2 diabetes mellitus: practical approaches for primary care physicians. J Am Osteopath Assoc. 2012;111(5 suppl 4):S3-S12.

3. Ramos W, López T, Revilla L, More L, Huamaní M, Pozo M. Resultados de la vigilancia epidemiológica de diabetes mellitus en hospitales notificantes del Perú, 2012. Rev Peru Med Exp Salud Publica. 2014;31(1):9-15.

4. Encuesta Nacional de Salud y Nutrición. Diabetes en adultos: urgente mejorar la atención y el control. 2012.

5. Estrada K, Aukrust I, Bjorkhaug L, Burtt N, Mercader J, García-Ortiz H et al. Association of a Low-Frequency Variant inHNF1AWith Type 2 Diabetes in a Latino Population. JAMA. 2014;311(22):2305.

6. Sell-Lluveras JL, Domínguez-Ihosvani M. Guía práctica para el diagnóstico y el tratamiento del síndrome del pie diabético. Rev Cubana Endocrinol [Internet]. 2001:12( 3);188-189. [citado 2017 May 31]; Disponible en: http://scielo.sld.cu/scielo.php?script=sci_arttext\&pi$d=S 1561-29532001000300008 \&$ Ing =es.

7. Alcántara-Pío W, Flores R, Garmendia F. Prevalencia y Riesgo de Amputación en Pacientes con Pie Diabético. In Anales de la Facultad de Medicina. 2017:60(3);159164.

8. Fernández MT, Montequín JIF. Manual para la prevención, diagnóstico y tratamiento del pie diabético. Revista Cubana de Angiología y Cirugía Vascular. 2009:10(1);4296.

9. Rivero-Fernández F, Vejerano-García P, González-González F. Clasificación actualizada de los factores de riesgo del pie diabético. AMC [Internet]. 2005:9(1);64-72. Feb [citado 2017 May 31]; Disponible en: http://scielo.sld.cu/scielo.php?script=sci_arttext\&pid=S1025-02552005000100008\&lng=es.
10. OMS. Informe mundial sobre la diabetes. Resumen de orientación. 2014.

11. Cortez-Salvatierra KX. Factores de riesgo del pie diabético en paciente de 45 a 70 años en el Hospital" Abel Gilbert Pontón" durante el período de enero del 2014 a diciembre del 2014. 2015.

12. Polit $D$, Beck $C$. Essentials of nursing research. Philadelphia: Lippincott; 2006.

13. George D, Mallery P. SPSS for Windows step by step: A simple guide and reference 11.0 update. 2003:4.

14. Secretaria de Salud. Reglamento de la Ley General de Salud en Materia de Investigación para la salud. México, D.F. 1988.

15. Ibáñez JA, Pairet JG, Unanue S, Gelabert RD. Identificación del riesgo de pie diabético y factores asociados. Enfermería Clínica. 2005:15(5);283-6.

16. Alvarez SE, Mena BK, Faget CO, et al. El pie de riesgo de acuerdo con su estratificación en pacientes con diabetes mellitus. Revista Cubana de Endocrinología. 2015: 26 (2);158-71.

17. Collar MV. Identificación del pie en riesgo de las personas con diabetes (Doctoral dissertation, Tesis de Ciencias Médicas). San Martín de la plata-Argentina. Universidad Nacional de la Plata. 2010.

18. González de la Torre H, Perdomo EP, Lorenzo Q, Luana M, Mosquera AF. Estratificación de riesgo en pie diabético. Gerokomos, 2010:21(4);172-82. 